\title{
The Gaia-ESO Survey: Probes of the inner disk abundance gradient ${ }^{\star}, \star \star$
}

\author{
H. R. Jacobson ${ }^{1}$, E. D. Friel ${ }^{2}$, L. Jílková ${ }^{3}$, L. Magrini ${ }^{4}$, A. Bragaglia ${ }^{5}$, A. Vallenari' ${ }^{6}$, M. Tosi ${ }^{5}$, S. Randich $^{4}$, \\ P. Donati ${ }^{5,7}$, T. Cantat-Gaudin ${ }^{6,8}$, R. Sordo ${ }^{6}$, R. Smiljanic ${ }^{9}$, J. C. Overbeek ${ }^{2}$, G. Carraro ${ }^{10}$, G. Tautvaišiené ${ }^{11}$, \\ I. San Roman ${ }^{12}$, S. Villanova ${ }^{13}$, D. Geisler ${ }^{13}$, C. Muñoz ${ }^{13}$, F. Jiménez-Esteban ${ }^{14,15}$, B. Tang ${ }^{13}$, G. Gilmore ${ }^{16}$, \\ E. J. Alfaro ${ }^{17}$, T. Bensby ${ }^{18}$, E. Flaccomio ${ }^{19}$, S. E. Koposov ${ }^{16,26}$, A. J. Korn ${ }^{20}$, E. Pancino ${ }^{4,21}$, A. Recio-Blanco ${ }^{22}$, \\ A. R. Casey ${ }^{16}$, M. T. Costado ${ }^{17}$, E. Franciosini ${ }^{4}$, U. Heiter ${ }^{20}$, V. Hill ${ }^{22}$, A. Hourihane ${ }^{16}$, C. Lardo ${ }^{23}$, P. de Laverny ${ }^{22}$, \\ J. Lewis ${ }^{16}$, L. Monaco ${ }^{24}$, L. Morbidelli ${ }^{4}$, G. G. Sacco ${ }^{4}$, S. G. Sousa ${ }^{25}$, C. C. Worley ${ }^{16}$, and S. Zaggia ${ }^{6}$ \\ (Affiliations can be found after the references)
}

Received 27 October 2015 / Accepted 4 May 2016

\begin{abstract}
Context. The nature of the metallicity gradient inside the solar circle $\left(R_{\mathrm{GC}}<8 \mathrm{kpc}\right)$ is poorly understood, but studies of Cepheids and a small sample of open clusters suggest that it steepens in the inner disk.

Aims. We investigate the metallicity gradient of the inner disk using a sample of inner disk open clusters that is three times larger than has previously been studied in the literature to better characterize the gradient in this part of the disk.

Methods. We used the Gaia-ESO Survey (GES) [Fe/H] values and stellar parameters for stars in 12 open clusters in the inner disk from GES-UVES data. Cluster mean $[\mathrm{Fe} / \mathrm{H}]$ values were determined based on a membership analysis for each cluster. Where necessary, distances and ages to clusters were determined via comparison to theoretical isochrones.

Results. The GES open clusters exhibit a radial metallicity gradient of $-0.10 \pm 0.02 \mathrm{dex} \mathrm{kpc}^{-1}$, consistent with the gradient measured by other literature studies of field red giant stars and open clusters in the range $R_{\mathrm{GC}} \sim 6-12 \mathrm{kpc}$. We also measure a trend of increasing [Fe/H] with increasing cluster age, as has also been found in the literature.

Conclusions. We find no evidence for a steepening of the inner disk metallicity gradient inside the solar circle as earlier studies indicated. The age-metallicity relation shown by the clusters is consistent with that predicted by chemical evolution models that include the effects of radial migration, but a more detailed comparison between cluster observations and models would be premature.
\end{abstract}

Key words. Galaxy: formation - Galaxy: disk - Galaxy: abundances - stars: abundances

\section{Introduction}

Ever since the seminal work of Janes (1979), open clusters have been used to trace the metallicity distribution in the Galactic disk. In that work, Janes found that the distribution of metallicity $([\mathrm{Fe} / \mathrm{H}])$ with Galactocentric distance $\left(R_{\mathrm{GC}}\right)$ was consistent with a gradient with metallicity decreasing with increasing distance from the Galactic center at a rate of $-0.05 \mathrm{dex} \mathrm{kpc}^{-1}$. Such a distribution has been shown in chemical evolution models to represent the inside-out growth of the Galactic disk (e.g., Chiappini et al. 1997). The term "gradient" has come to be used as short hand to refer to the (azimuthally averaged) change of $[\mathrm{Fe} / \mathrm{H}]$ as a function of $R_{\mathrm{GC}}$ and has generally been described as a linear function, as in Janes (1979). However, it

\footnotetext{
* Based on data products from observations made with ESO Telescopes at the La Silla Paranal Observatory under programme ID 188.B-3002 and 193.B-0936. These data products have been processed by the Cambridge Astronomy Survey Unit (CASU) at the Institute of Astronomy, University of Cambridge, and by the FLAMES/UVES reduction team at INAF/Osservatorio Astrofisico di Arcetri. These data have been obtained from the Gaia-ESO Survey Data Archive, prepared and hosted by the Wide Field Astronomy Unit, Institute for Astronomy, University of Edinburgh, which is funded by the UK Science and Technology Facilities Council.

$\star \star$ Full Table 2 is only available at the CDS via anonymous ftp to cdsarc.u-strasbg.fr (130.79.128.5) or via

http://cdsarc.u-strasbg.fr/viz-bin/qcat?]/A+A/591/A37
}

has been pointed out several times over the decades that the metallicity distribution with $R_{\mathrm{GC}}$ shown by open clusters and other stellar populations can be described by other functions, as well (e.g., Twarog et al. 1997; Andrievsky et al. 2002). Indeed, as the number of clusters studied by high-resolution spectroscopy has grown over the past decade, it now seems clear that the form of the radial dependence on mean metallicity in the disk changes with Galactocentric radius. While a linearly decreasing function may well describe the change in $[\mathrm{Fe} / \mathrm{H}]$ as a function of $R_{\mathrm{GC}}$ through the solar neighborhood out to $R_{\mathrm{GC}} \sim 10-13 \mathrm{kpc}$, the clusters in the outer disk have roughly constant $[\mathrm{Fe} / \mathrm{H}] \sim-0.3$ and remarkably small dispersion (e.g., Heiter et al. 2014).

Much work has been done in recent years to better characterize the gradient in the outer disk, and the nature of the transition between the inner and outer disk (e.g., Carraro et al. 2007; Yong et al. 2012; Bragaglia et al. 2008; Jacobson et al. 2011; Cantat-Gaudin et al. 2016). It can be said, however, that the gradient in the inner disk has received comparatively less attention. This is not surprising given that the line of sight toward the Galactic center is much more crowded, confused, and extincted. Nevertheless, this is an important part of the Galaxy where the bulge/bar meets both the thin and thick disks.

There is some evidence that the nature of the gradient changes inside the solar circle. Some of the first evidence came from Cepheids: Andrievsky et al. (2002) found that the gradient 
inside $R_{\mathrm{GC}} \sim 7-8 \mathrm{kpc}$ was steeper than that outside the solar circle. Recent work by Genovali et al. $(2013,2014)$ has reinforced the increase in $[\mathrm{Fe} / \mathrm{H}]$ seen in the inner disk to super solar metallicities of $\sim 0.4$ dex. The Cepheid sample of Martin et al. (2015), which extends to as close as $3 \mathrm{kpc}$ from the Galactic center, indicates that $0.4 \mathrm{dex}$ is the upper bound of the metallicity range in the inner disk, and the gradient plateaus at this level inside of $R_{\mathrm{GC}}=5 \mathrm{kpc}$. In contrast to the evidence from Cepheids, a study of inner disk red clump stars has found no evidence for a gradient at all: red clump stars spanning $R_{\mathrm{GC}} \sim 4.5-6.5 \mathrm{kpc}$ along a line of sight towards the Galactic center have a mean $[\mathrm{Fe} / \mathrm{H}]=+0.17$ with no sign of a change with $R_{\mathrm{GC}}$ (Hill et al. 2012).

Regarding the open cluster population, the number of confirmed clusters inside $R_{\mathrm{GC}} \sim 7 \mathrm{kpc}$ that we could use to probe the inner disk gradient is small relative to that in the solar neighborhood and beyond. Many works have noted the dearth of old $(>1 \mathrm{Gyr}$ ) clusters in the inner region (see, e.g., Bonatto et al. 2006) and while new surveys in the infrared are revealing promising candidates for future study (e.g., Froebrich et al. 2007; Borissova et al. 2014), many of these candidate clusters are embedded and highly obscured. Relatively few have fundamental parameters determined, and of those even fewer are accessible to traditional optical techniques for abundance analysis. However, there have been efforts to study the known open clusters in the inner disk specifically to determine whether the older population of open clusters shows the same steepening of the gradient that is seen in the younger Cepheids. With their sample of three clusters all inside $R_{\mathrm{GC}} \sim 7 \mathrm{kpc}$, along with a selection of clusters from the literature, Magrini et al. (2010) did indeed find evidence that the gradient rises more sharply towards the galactic center. While this work greatly increased the number of inner disk open clusters subject to detailed chemical abundance study, the inner disk cluster sample that has been well studied is still small relative to that in the solar neighborhood and beyond.

In the last few years, a number of spectroscopic surveys have been planned and begun, aimed at investigating and constraining in great detail the chemo-dynamical evolution of the Milky Way: for example, the extentions to SDSS (York et al. 2000) SEGUE (Yanny et al. 2009) and APOGEE (Majewski et al. 2015); RAVE (Steinmetz et al. 2006); the Gaia-ESO Survey (Gilmore et al. 2012; Randich et al. 2013); GALAH (De Silva et al. 2015). Several multi-object spectrographs have been designed and/or built that will also carry out their own spectroscopic surveys: LAMOST (Zhao et al. 2006; Cui et al. 2012); WEAVE (Dalton et al. 2012; Balcells 2014); MOONS (Cirasuolo et al. 2011); 4MOST (de Jong et al. 2014). In particular, the SDSS III APOGEE Survey (Ahn et al. 2014; Majewski et al. 2015) has done much to increase our picture of the metallicity distribution of the disk, based on near-infrared high-resolution spectroscopy of red giant stars. While the APOGEE Survey includes many open clusters, their sample predominantly has objects located at $R_{\mathrm{GC}} \gtrsim 7$ kpc (Frinchaboy et al. 2013; Cunha et al. 2016), and in some cases only a few members per cluster were observed. Instead, insight into the inner disk metallicity distribution comes from a study of APOGEE field stars, which found that the gradient of $\sim-0.09 \mathrm{dex} \mathrm{kpc}^{-1}$ in the Solar neighborhood transitions around $R_{\mathrm{GC}}=6 \mathrm{kpc}$, becoming shallower interior to that value (Hayden et al. 2014, 2015).

The Gaia-ESO Survey (GES) is a large-scale, highresolution spectroscopic survey being carried out with the FLAMES instrument on the VLT. In addition to probing field stars in all major stellar components of the Milky Way, the survey includes a large number of open clusters spanning a wide range in age and location in the Galactic disk (Gilmore et al. 2012, Randich et al. 2013). Stars of all evolutionary states are observed in GES, from pre-main sequence stars to red giants, and elements from all groups of the Periodic Table, from Li to the neutron-capture species, are measurable in the FLAMES spectra. Regarding open clusters, with the complementary capabilities of the GIRAFFE and UVES spectographs, GES stellar samples can range from $10^{2}$ to $10^{3}$ members per cluster (e.g., Donati et al. 2014b; Cantat-Gaudin et al. 2014; Frasca et al. 2015; Prisinzano et al. 2016) allowing not only for robust average measures of cluster chemistry and dynamics, but also their variations as a function of stellar evolutionary state (Smiljanic et al. 2016).

Though the survey is far from complete, the first internal data releases from GES (data releases iDR1, iDR2/3, iDR4) span roughly 2.5 out of five years of observations and provide an opportunity to explore the form of the abundance gradient in the inner disk from high-resolution spectroscopy of both open clusters and field stars. Complementary studies of the thin and thick disk gradients and age-metallicity relationships using GES iDR1 data for field stars have appeared in Bergemann et al. (2014), Recio-Blanco et al. (2014) and Mikolaitis et al. (2014). Bergemann et al. (2014) used UVES data of field FGK stars to explore the age-metallicity relationships and gradients in the thin and thick disk populations. To obtain ages, they limited their sample to unevolved stars and so probed only the solar neighborhood at distances of $7<R_{\mathrm{GC}}<9 \mathrm{kpc}$. Recio-Blanco et al. (2014) used the lower resolution GIRAFFE spectra of FGK stars in the first data release to study the kinematic and spatial gradients of the thin and thick disk populations over the range $5<$ $R_{\mathrm{GC}}<12 \mathrm{kpc}$. Mikolaitis et al. (2014) used a subset of the Recio-Blanco et al. (2014) sample and the distances calculated therein that spanned a range of $4<R_{\mathrm{GC}}<12 \mathrm{kpc}$ and found a rather shallow metallicity gradient for the thin disk $(\sim-0.03$

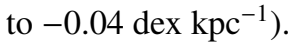

While current sample sizes of field stars in spectroscopic surveys such as GES and APOGEE reveal much about the state of our Galaxy, open clusters within these surveys are complementary tracers of the chemistry of the thin disk because their ages and distances can be precisely determined, they span a wide range in age and location within the Galaxy, and their chemical abundances can be robustly measured from large samples of stellar members. In this work, we utilize the iDR4 GES results obtained from UVES observations of stars in 12 intermediate-aged ( $>100 \mathrm{Myr},<3 \mathrm{Gyr}$ ) open clusters. These 12 are from a total of 26 open clusters included in iDR4. These results are placed in the context of those of other inner disk populations to explore the nature of the abundance gradient in this relatively understudied region of the Galaxy. This paper is organized as follows: Sect. 2 presents the GES observations used in this work; Sect. 3 presents the discussion of the inner disk metallicity gradient and Sect. 4 compares it to chemical evolution models. A summary and conclusions are available in Sect. 5.

\section{GES data}

The Gaia-ESO survey began on 31 December 2011. The survey makes use of the FLAMES instrument with both GIRAFFE and the fiber link to UVES. Here, we used only the UVES spectra obtained with the $580 \mathrm{~nm}$ setup, which have spectral resolution $R \equiv \lambda / \Delta \lambda=47000$, signal-to-noise $(S / N) \gtrsim 50$ and span 4760-6840 $\AA$. UVES data were processed with the GES pipeline as described in Sacco et al. (2014). Stellar parameters and abundances for FGK-type stars observed with UVES 
Table 1. Cluster parameters.

\begin{tabular}{llllllllll}
\hline \hline Cluster & $l$ & $b$ & $\begin{array}{l}\text { Age } \\
\left({ }^{\circ}\right)\end{array}$ & $\begin{array}{l}{[\mathrm{Fe} / \mathrm{H}]} \\
(\mathrm{o})\end{array}$ & $\begin{array}{l}\mathrm{RV} \\
(\mathrm{Gyr})\end{array}$ & \# stars & $\begin{array}{l}R_{\mathrm{GC}} \\
(\mathrm{kpc})\end{array}$ & $\begin{array}{l}z \\
(\mathrm{pc})\end{array}$ & Ref. \\
\hline Berkeley 44 & 53.2 & +3.33 & $1.6 \pm 0.3$ & $+0.17 \pm 0.04$ & $-8.7 \pm 0.7$ & 4 & $6.91 \pm 0.12$ & $128 \pm 17$ & This study \\
Berkeley 81 & 34.51 & -2.07 & $0.86 \pm 0.10$ & $+0.21 \pm 0.06$ & $+48.3 \pm 0.6$ & 13 & $5.49 \pm 0.10$ & $-126 \pm 7$ & 1,2 \\
NGC 2516 & 273.8 & -15.8 & $0.16 \pm 0.04$ & $-0.06 \pm 0.05$ & $+23.6 \pm 1.0$ & 15 & $7.98 \pm 0.01$ & $-97 \pm 4$ & 3 \\
NGC 3532 & 289.6 & 1.35 & $0.30 \pm 0.10$ & $-0.03 \pm 0.02$ & $+4.8 \pm 1.4$ & 2 & $7.85 \pm 0.01$ & $12 \pm 1$ & 4 \\
NGC 4815 & 303.63 & -2.10 & $0.57 \pm 0.07$ & $-0.03 \pm 0.06$ & $-29.6 \pm 0.5$ & 5 & $6.94 \pm 0.04$ & $-95 \pm 6$ & 5 \\
NGC 6005 & 325.8 & -3.00 & $1.20 \pm 0.30$ & $+0.16 \pm 0.02$ & $-24.1 \pm 1.34$ & 12 & $5.97 \pm 0.34$ & $-141 \pm 26$ & 6 \\
NGC 6633 & 36.0 & 8.3 & $0.63 \pm 0.10$ & $-0.05 \pm 0.06$ & $-28.8 \pm 1.5$ & 11 & $7.71 \pm 0.01$ & $52 \pm 2$ & 7 \\
NGC 6705 & 27.31 & -2.78 & $0.30 \pm 0.05$ & $+0.08 \pm 0.05$ & $+34.9 \pm 1.6$ & 27 & $6.33 \pm 0.16$ & $-95 \pm 10$ & 8 \\
NGC 6802 & 55.3 & 0.92 & $1.0 \pm 0.1$ & $+0.10 \pm 0.02$ & $+11.9 \pm 0.9$ & 8 & $6.96 \pm 0.07$ & $36 \pm 3$ & This study \\
Pismis 18 & 308.2 & 0.30 & $1.2 \pm 0.4$ & $+0.11 \pm 0.02$ & $-27.5 \pm 0.7$ & 6 & $6.85 \pm 0.17$ & $12 \pm 2$ & 6 \\
Trumpler 20 & 301.48 & +2.22 & $1.50 \pm 0.15$ & $+0.10 \pm 0.05$ & $-40.2 \pm 1.3$ & 42 & $6.86 \pm 0.01$ & $136 \pm 4$ & 9 \\
Trumpler 23 & 328.8 & -0.50 & $0.8 \pm 0.1$ & $+0.14 \pm 0.03$ & $-61.3 \pm 0.9$ & 10 & $6.25 \pm 0.15$ & $-18 \pm 2$ & This study \\
\hline
\end{tabular}

References. References for cluster ages, $R_{\mathrm{GC}}$ and $z$ distances: (1) Donati et al. (2014a); (2) Magrini et al. (2015); (3) Sung et al. (2002); (4) Clem et al. (2011); (5) Friel et al. (2014); (6) Piatti et al. (1998); (7) Jeffries et al. (2002); (8) Cantat-Gaudin et al. (2014); (9) Donati et al. (2014b).

were determined by up to 14 independent groups, and homogenized in a process described in Smiljanic et al. (2014). Results from working groups specializing in different stellar types were then combined to result in a set of recommended parameters utilized by the GES collaboration (Hourihane et al., in prep.). These recommended parameters, available in the "recommendedastroanalysis" table within GESiDR4Final, were used in this work.

Analysis has now been completed and the data products for all survey observations taken before July 2014 have been released to the consortium as GESviDR4Final. This data release included some updated analysis methods and homogenization across multiple working groups. As a result, this release included newly determined stellar parameters and abundances for the previously analyzed objects, so the parameters presented here differ slightly from those in earlier GES publications.

Twelve intermediate-aged clusters inside the solar circle (for this work we adopted $R_{\mathrm{GC}}=8.0 \mathrm{kpc}$ for the Sun) have been targeted with UVES observations and included in GESviDR4Final (Table 1). Of these, results from earlier internal data releases were published for the clusters Trumpler 20, NGC 4815, NGC 6705 (M 11) and Berkeley 81. References for these works are given in Table 1. For these objects, we adopted the ages and distances determined from those published papers. For five of the remaining eight clusters we used values from the literature as given in Table 1. Three of these clusters are nearby and have distances that rest on Hipparcos measurements (NGC 2516, NGC 3532, and NGC 6633). Two others (NGC 6005 and Pismis 18) have parameters that rely on traditional methods of fitting isochrones, but with models that are consistent with the metallicities derived here; we explore the impact of errors on their distances below. For the remaining three clusters, we estimated new cluster ages, distances, and $E(B-V)$ values taking into account the new GES metallicities, which are significantly higher than previously assumed metallicities (Be 44 and NGC 6802), or for which we already had analysis underway (Trumpler 23, Overbeek et al., in prep.). Analysis for these clusters was intended only to give preliminary values that primarily take into account the revised metallicity and membership information from GES; a more complete treatment and redetermination of cluster parameters will be presented in future publications (e.g., Tang et al., in prep. for NGC 6802). For these clusters, we used radial velocities measured from both UVES and GIRAFFE observations in the cluster field to determine estimates of cluster systemic velocities. First, a mean radial velocity was calculated from all stars in the cluster field. Probable radial velocity members were chosen as those stars within approximately 2 sigma about the mean velocity. Using this indication of membership in conjuction with published photometry (Janes \& Hoq 2011, for Be 44 and NGC 6802 and Carraro et al. 2006, for Trumpler 23), we fit PARSEC isochrones (Bressan et al. 2012) at the derived GES metallicities (see below), to obtain estimates of cluster reddening, distance, and age which are summarized in Table 1.

For clusters available in earlier GES releases, we determined membership independently of those previously published. Not only have the recommended parameters and abundances varied slightly with each data release as a consequence of the calibration process, but in the case of some clusters, additional fields were observed and include more cluster members than were available in iDR1 and iDR2/3. Mean cluster metallicities were based on an evaluation of individual star cluster membership using radial velocities and abundances from UVES spectra in GESviDR4Final. We used the radial velocities in combination with the derived $[\mathrm{Fe} / \mathrm{H}]$ values from UVES observations as a guide for determining membership; cluster members clump tightly in a diagram of radial velocity versus $[\mathrm{Fe} / \mathrm{H}]$, aiding the elimination of potential non-member stars near the cluster velocity. The majority of $[\mathrm{Fe} / \mathrm{H}]$ values came from evolved stars in the red clump or along the giant branch, but for the closest clusters NGC 2516, 3532 and 6633, include determinations from dwarf stars as well. The resulting mean cluster abundances and standard deviations about the mean and radial velocities, based on these likely members, are given in Table 1 . The GES ID, coordinates, and the GES recommended parameters for all stars considered cluster members and used in forming the mean metallicities are given in Table 2.

The location of these inner disk GES clusters relative to the plane of the Milky Way disk is shown in the top panel of Fig. 1. All clusters are within $\sim 150 \mathrm{pc}$ of the plane, and appear to be thin disk objects. The bottom panel of Fig. 1 shows the range of cluster age as a function of $R_{\mathrm{GC}}$. Clusters at similar $R_{\mathrm{GC}}$ can vary in age by as much as 1 Gyr.

\section{Inner disk metallicity gradient}

With nine open clusters inside $R_{\mathrm{GC}}=7 \mathrm{kpc}$, the GES sample is a factor of three times larger than previous homogeneous samples in this part of the Galactic disk (Magrini et al. 2010) and allows us to see more clearly the trend of metallicity inside the solar 
Table 2. Parameters for cluster members.

\begin{tabular}{|c|c|c|c|c|c|c|c|c|c|c|}
\hline Cluster & $\begin{array}{l}\text { Star } \\
\text { GES ID }\end{array}$ & $\begin{array}{l}\text { RA (J2000) } \\
\text { (deg) }\end{array}$ & $\begin{array}{l}\text { Dec (J2000) } \\
(\mathrm{deg})\end{array}$ & $\begin{array}{l}{[\mathrm{Fe} / \mathrm{H}]} \\
(\mathrm{dex})\end{array}$ & $\begin{array}{l}e([\mathrm{Fe} / \mathrm{H}]) \\
(\operatorname{dex})\end{array}$ & $\begin{array}{l}T_{\text {eff }} \\
(\mathrm{K})\end{array}$ & $\begin{array}{l}e\left(\mathrm{~T}_{\text {eff }}\right) \\
(\mathrm{K})\end{array}$ & $\begin{array}{l}\log g \\
(\operatorname{dex})\end{array}$ & $\begin{array}{l}e(\log g) \\
(\operatorname{dex})\end{array}$ & $\begin{array}{l}\mathrm{RV} \\
\left(\mathrm{km} \mathrm{s}^{-1}\right)\end{array}$ \\
\hline Berkeley44 & $19170732+1930555$ & 289.2805000 & 19.5154167 & 0.11 & 0.14 & 4998 & 233 & 3.13 & 0.55 & -7.7 \\
\hline Berkeley44 & $19170911+1933256$ & 289.2879583 & 19.5571111 & 0.20 & 0.12 & 4943 & 182 & 3.06 & 0.44 & -9.1 \\
\hline Berkeley44 & $19171388+1933333$ & 289.3078333 & 19.5592500 & 0.22 & 0.14 & 4996 & 181 & 2.79 & 0.46 & -9.1 \\
\hline Berkeley44 & $19172208+1933254$ & 289.3420000 & 19.5570556 & 0.15 & 0.13 & 4880 & 222 & 2.77 & 0.56 & -8.7 \\
\hline Berkeley81 & $19013537-0028186$ & 285.3973750 & -0.4718333 & 0.27 & 0.13 & 5167 & 142 & 3.30 & 0.30 & 47.5 \\
\hline Berkeley81 & $19013631-0027447$ & 285.4012917 & -0.4624167 & 0.25 & 0.11 & 4991 & 138 & 2.79 & 0.30 & 47.6 \\
\hline Berkeley81 & $19013651-0027021$ & 285.4021250 & -0.4505833 & 0.22 & 0.12 & 4939 & 127 & 3.14 & 0.24 & 48.9 \\
\hline Berkeley81 & $19013910-0027114$ & 285.4129167 & -0.4531667 & 0.12 & 0.10 & 5012 & 117 & 2.96 & 0.24 & 48.8 \\
\hline Berkeley81 & $19013997-0028213$ & 285.4165417 & -0.4725833 & 0.32 & 0.10 & 4970 & 149 & 3.27 & 0.27 & 48.6 \\
\hline Berkeley81 & $19014004-0028129$ & 285.4168333 & -0.4702500 & 0.26 & 0.10 & 4940 & 127 & 2.77 & 0.24 & 48.7 \\
\hline
\end{tabular}

Notes. The complete table is available at the CDS. The first few lines are shown here as a guide to its contents.

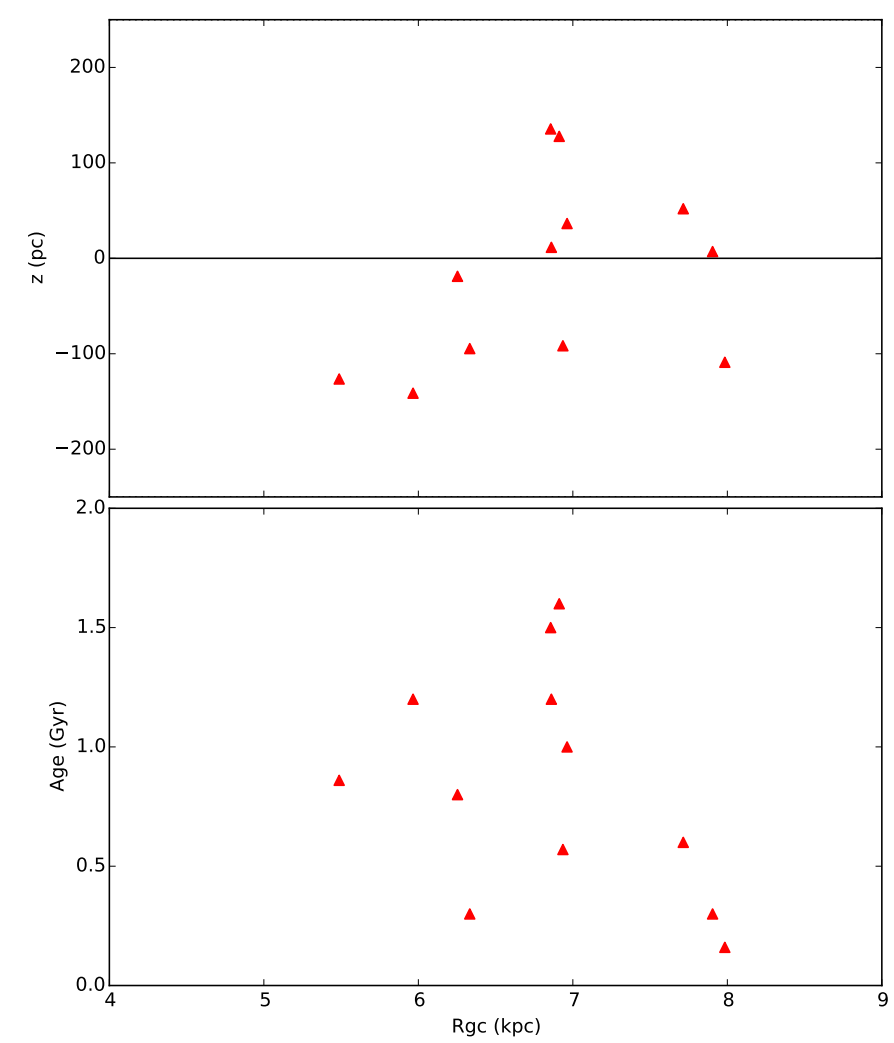

Fig. 1. Top panel: locations of the GES open clusters (red triangles) relative to the Galactic plane ( $z=0$, solid line), as a function of their Galactocentric radii. All clusters lie within $150 \mathrm{pc}$ of the mid-plane, and thus are members of the thin disk. Bottom panel: cluster ages (in Gyr) plotted as a function of $R_{\mathrm{GC}}$.

radius. As shown in Fig. 2, the sample of 12 GES clusters (in red triangles) shows a steady decrease in $[\mathrm{Fe} / \mathrm{H}]$ with increasing Galactocentric distance over the range of $R_{\mathrm{GC}} \sim 5.5-8 \mathrm{kpc}$. The vertical error bars represent the standard deviations about the mean abundance determined from those stars judged to be members, and given in Table 1. As discussed in Magrini et al. (2014) and Magrini et al. (2015), in light of the highly uniform abundance scale for the GES determinations and the internal chemical homogeneity of the clusters, the differences in metallicity seen among the GES clusters are reliable and significant. The current sample reinforces this fact; as can be seen from the stellar parameters given in Table 2, typical uncertainties in $[\mathrm{Fe} / \mathrm{H}]$ for individual stars are 0.10 dex. The rms deviations about the

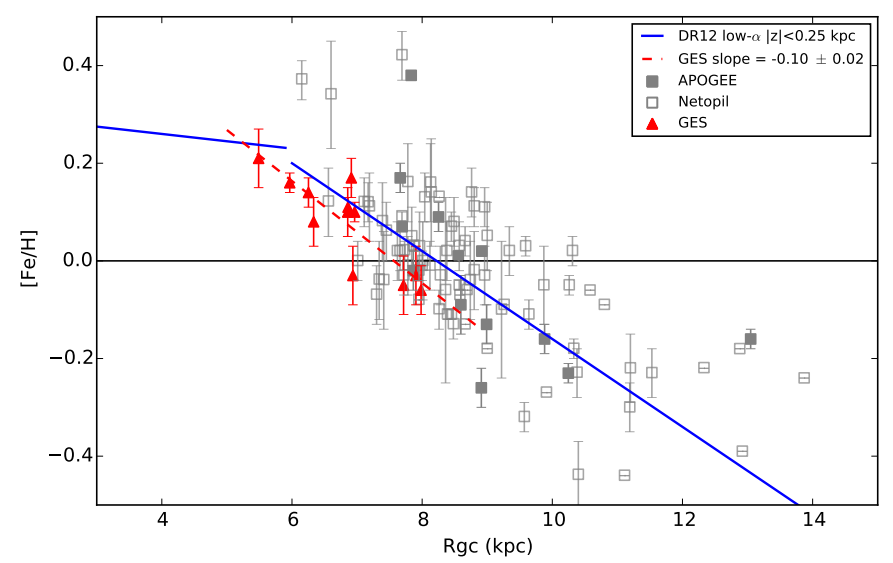

Fig. 2. Metallicity gradient as shown by the GES open clusters (red triangles) in comparison to literature studies. The open cluster literature compilation of Netopil et al. (2016) is shown as open gray squares, while clusters observed by the APOGEE survey (Frinchaboy et al. 2013) are filled gray squares. The solid blue line shows the metallicity gradient as determined by APOGEE field giant stars within $250 \mathrm{pc}$ of the Galactic mid-plane (Hayden et al. 2014). The red dashed line is least-squares fit to the GES sample, with the value of the slope given in the caption. $[\mathrm{Fe} / \mathrm{H}]=0$ is indicated by the solid black line, to guide the eye.

cluster means, on the other hand, are much smaller, from 0.02 to $0.06 \mathrm{dex}$, indicating that there is no evidence of an internal spread in abundance within each cluster. There is a clear decrease in mean abundance from the innermost point sampled, at $R_{\mathrm{GC}}=5.5 \mathrm{kpc}$, out to the clusters close to the solar neighborhood. A linear regression analysis on the GES sample shows a metallicity gradient with a slope of $-0.10 \pm 0.02 \mathrm{dex} \mathrm{kpc}^{-1}$.

In addition to this clear trend, however, there is significant variation in $[\mathrm{Fe} / \mathrm{H}]$ around $R_{\mathrm{GC}}=7 \mathrm{kpc}$. The 5 clusters at $R_{\mathrm{GC}}=7 \mathrm{kpc}$ cover a range of $0.2 \mathrm{dex}$, from Be 44 at $[\mathrm{Fe} / \mathrm{H}]=$ $+0.17 \pm 0.04$ to NGC 4815 , with $[\mathrm{Fe} / \mathrm{H}]=-0.03 \pm 0.06$, well in excess of the scatter about the mean cluster metallicities. Since stars in these two clusters have similar atmospheric parameters and were analyzed homogeneously, this dispersion in $[\mathrm{Fe} / \mathrm{H}]$ is likely real. Of the five clusters around $R_{\mathrm{GC}}=7 \mathrm{kpc}$, two have $l \sim 54^{\circ}$, and the other three cluster around $l \sim 305^{\circ}$ (Table 1). However these two groups do not also cluster together in metallicity: the $[\mathrm{Fe} / \mathrm{H}]$ dispersion at each Galactic longitude is 0.07 and 0.13 dex, respectively.

As mentioned previously, this homogeneous GES cluster sample explores the inner part of the disk with considerably larger statistics than in previous studies. More clusters will be 
available in future GES releases, sampling also the outer parts of the disk. At this stage, in order to study the behavior of the metallicity distribution across a wider range of Galactocentric distances, we also consider the large compilation of high-resolution open cluster metallicities in Netopil et al. (2016). This compilation is an update to that of Heiter et al. (2014), who determined weighted average $[\mathrm{Fe} / \mathrm{H}]$ values for clusters based on rigorous selection criteria over a limited range of atmospheric parameters of literature measures. Though the numbers of open clusters observed homogeneously as part of large spectroscopic surveys (e.g., in GES; APOGEE - Frinchaboy et al. 2013; LAMOST Zhang et al. 2015) is growing, the Heiter et al. (2014) sample and its update is currently the closest one can come to a uniform sample formed from inhomogeneous literature measurements. Eight clusters in the current work are also in Netopil et al. (2016); we use the $[\mathrm{Fe} / \mathrm{H}]$, age and $R_{\mathrm{GC}}$ values given in Table 1 for the clusters in common.

Figure 2 shows the metallicity distribution with $R_{\mathrm{GC}}$ for both the GES inner disk clusters (red triangles), and the Netopil et al. (2016) sample (open gray squares). We note that though clusters beyond $R_{\mathrm{GC}}=14 \mathrm{kpc}$ have been studied, we limit our discussion to objects inside this limit, as the gradient appears to change around this Galactocentric radius (e.g., Yong et al. 2005, 2012; Carraro et al. 2007; Pancino et al. 2010; Andreuzzi et al. 2011; Donati et al. 2015; Netopil et al. 2016; see also Twarog et al. 1997). For comparison, we also plot open clusters observed as part of the APOGEE Survey (Frinchaboy et al. 2013; filled gray squares) ${ }^{1}$.

The linear gradient traced by the GES clusters, $-0.10 \pm$ $0.02 \mathrm{dex} \mathrm{\textrm {kc } ^ { - 1 }}$ is shown as the dashed red line. The gradient described by the APOGEE cluster sample is just as steep, if not steeper; Frinchaboy et al. (2013) reported $-0.20 \pm 0.08 \mathrm{dex} \mathrm{kpc}^{-1}$ for clusters inside $R_{\mathrm{GC}}=10 \mathrm{kpc}^{2}$. However, these cluster samples overlap in a very limited region of $R_{\mathrm{GC}} \sim 7-8 \mathrm{kpc}$, and given the small sample sizes these slopes may not fully reflect the metallicity distribution in the inner disk. Fitting their larger literature sample Netopil et al. (2016) find a linear metal-

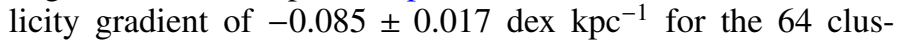
ters inside $9 \mathrm{kpc}$, a slope quite consistent with the relationship traced by the GES clusters. They note the strong influence of the three clusters with much higher metallicity at $R_{\mathrm{GC}} \sim 7 \mathrm{kpc}$ (NGC 6253, 6583, and 6791) and omitting those find a more modest slope of $-0.061 \pm 0.015 \mathrm{dex} \mathrm{kpc}^{-1}$.

We note that, while there is overlap in the distribution of metallicities of the various samples, the GES abundances appear on the lower boundary of the distribution of Netopil et al. (2016) and APOGEE values at the same $R_{\mathrm{GC}}$. The slightly lower $[\mathrm{Fe} / \mathrm{H}]$ values for the GES clusters around $R_{\mathrm{GC}} \sim 8 \mathrm{kpc}$ may indicate a $\sim 0.1$ dex zero-point offset between the GES and Netopil et al. (2016) metallicity scales. It is also possible that the small numbers of the GES clusters in the solar neighborhood shown here do not fully reflect its intrinsic abundance distribution, and a larger sample of clusters would include objects of solar metallicity. For the eight clusters in common between the GES and the Netopil et al. (2016) study, the difference in $[\mathrm{Fe} / \mathrm{H}]$ is only $0.04 \pm 0.05$ (s.d.), suggesting that the systematic differences are small. Given these caveats, the metallicity distribution of the GES clusters is consistent with that of the Netopil et al. (2016) sample.

\footnotetext{
1 We show only those APOGEE clusters in which more than one star was observed.

2 Frinchaboy et al. (2013) adopted $R_{\mathrm{GC}}=8.5 \mathrm{kpc}$ for the Sun, as opposed to $8.0 \mathrm{kpc}$ in this work.
}

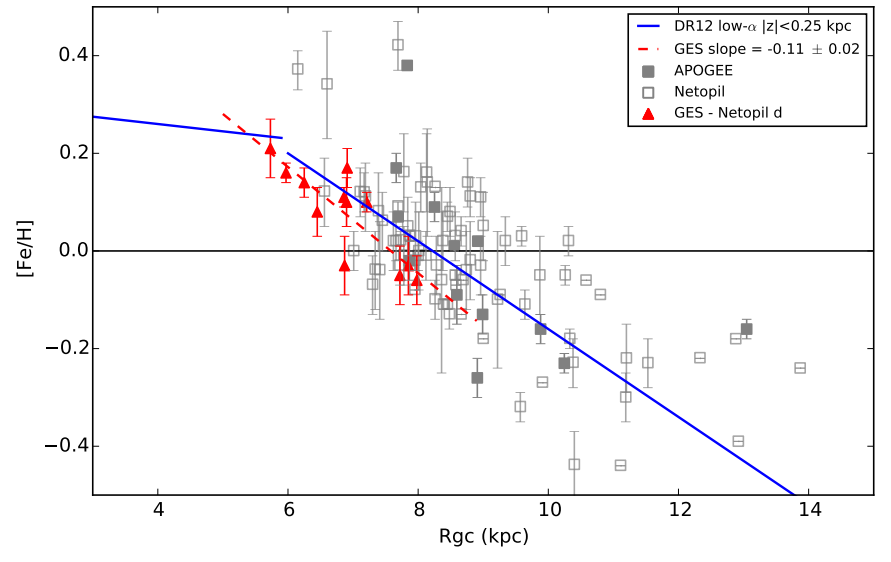

Fig. 3. Same as in Fig. 2, but with distances determined by Netopil et al. (2016) for GES clusters in their compilation. Note the magnitude of the GES cluster gradient has changed by $0.01 \mathrm{dex} \mathrm{kpc}^{-1}$.

Also shown in Fig. 2 is the gradient determined from $2 \times$ $10^{4}$ giant stars in the APOGEE Survey (Hayden et al. 2014). Specifically, we plot the gradient determined from a subsample of APOGEE stars with low $[\alpha / \mathrm{Fe}]$ measures, confined to within $250 \mathrm{pc}$ of the Galactic mid-plane (i.e., likely thin disk giant stars). As mentioned in the Introduction, Hayden et al. (2014) found that the metallicity gradient of -0.09 dex kpc $\mathrm{kp}^{-1}$ broke and became shallower inside $R_{\mathrm{GC}}=6 \mathrm{kpc}$. Only one GES object lies inside (though on the edge of) this break, and its $[\mathrm{Fe} / \mathrm{H}]$ value is consistent with both slopes shown by the APOGEE data.

Hayden et al. (2014) also traced the vertical abundance gradients in the disk, finding that they may be steeper in the inner Galaxy, with values of $-0.4 \mathrm{dex} \mathrm{kpc}^{-1}$ for the low $[\alpha / \mathrm{Fe}]$ stars inside $R_{\mathrm{GC}}=7 \mathrm{kpc}$. Figure 1 shows that the GES clusters reach at most $150 \mathrm{pc}$ from the Galactic plane, so the impact of this gradient, if it applied to the cluster population, would be at most $\sim 0.05$ dex. Correcting for this small effect, though, would serve to steepen the gradient by a small amount, to $-0.12 \mathrm{dex} \mathrm{kpc}^{-1}$ since the two innermost clusters are also among those most distant from the Galactic plane.

While the importance of homogeneous $[\mathrm{Fe} / \mathrm{H}]$ measures to the determination of the metallicity gradient cannot be overstated, distances must also be homogeneous. Given that the GES clusters and the Netopil et al. (2016) sample have different distance scales (though both adopt $R_{\mathrm{GC}}=8.0 \mathrm{kpc}$ for the Sun), we calculate the GES cluster metallicity gradient again adopting Netopil et al. (2016) $R_{\mathrm{GC}}$ values for objects in common. This is shown in Fig. 3. The gradient steepens slightly to $-0.11 \pm 0.02 \mathrm{dex} \mathrm{kpc}^{-1}$, as the Netopil et al. (2016) $R_{\mathrm{GC}}$ values are generally closer to the Sun than our values. We noted earlier that two of the clusters in the GES sample have larger than typical uncertainties in distance; these have little impact on the derived linear gradient. Changing their distances by the quoted errors changes the gradient by at most 0.01 . We conclude that, in general, the metallicity gradients shown by the GES sample, the Netopil et al. (2016) sample, and the APOGEE field star sample are consistent with one another.

The motivation of this work was to determine if the GES inner disk objects showed an indication of the metallicity gradient steepening inside the solar circle as previous studies have indicated (e.g., Genovali et al. 2014; Magrini et al. 2010). Given the consistency of the slopes of the different samples in Fig. 2 and Fig. 3, there is no indication that the open clusters' metallicity 


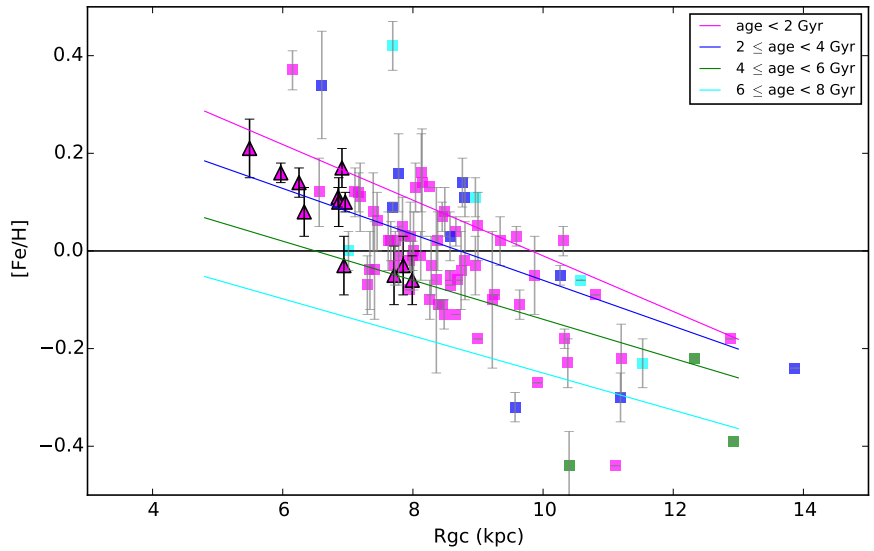

Fig. 4. GES open clusters (black-lined triangles) and Netopil et al (2016) clusters (filled squares), color-coded by age. The colored solid lines represent the metallicity gradients for different-aged stars in the chemical evolution model of Minchev et al. (2014). The gradient shown by the clusters is much steeper than that of the model.

gradient changes within the range of $R_{\mathrm{GC}} \sim 6-8 \mathrm{kpc}$, and in fact the APOGEE field star data indicate a shallower slope in the innermost region studied.

\section{Comparison to model predictions}

Open clusters have long been used to help constrain chemical evolution models of the Milky Way disk, through both the shape of their radial gradients and their evolution with time (e.g., Tosi 1982; Chiappini et al. 2001; Cescutti et al. 2007; Magrini et al. 2009). To date, the observational evidence for the time evolution of the gradient has been mixed. Generally, open cluster studies have found relatively little evidence of a change of the gradient with time, though there are some indications that the younger clusters have a slightly shallower distribution than the older clusters (a flattening of the gradient with time: Friel et al. 2002; Magrini et al. 2009; Carrera \& Pancino 2011; Andreuzzi et al. 2011). However, studies of other objects or combinations of different stellar populations have indicated the opposite. Stanghellini \& Haywood (2010), for example, found evidence that the gradient has grown steeper with time based on an analysis of disk planetary nebulae.

The chemical evolution model of Minchev et al. (2013, 2014) allows for the movement of star particles from their birthplace due to radial migration effects. (We take their work as an example; other dynamical models of Milky Way-like galaxies that trace stellar chemistry do exist, e.g., Roškar et al. 2008; Schönrich \& Binney 2009; Loebman et al. 2011; Bird et al. 2012; Kubryk et al. 2013.) Figure 4 shows the metallicity gradients predicted by their model for different-aged objects within $250 \mathrm{pc}$ of the Galactic plane (taken from Fig. 10 and Table 1 of Minchev et al. 2014). We also plot the GES and Netopil et al. (2016) open cluster samples color-coded with the same age ranges as the model gradients. The majority of open clusters are $<2$ Gyr old (magenta symbols), and show a steeper gradient than the model at all age ranges. We note also that the open clusters, almost regardless of age, do not align with the model predictions, either in the value of the metallicity or the dependance on age. It has been shown that open cluster metallicity does not correlate with cluster age as would arise from a general increase in the metallicity of the disk with time (e.g., Friel et al. 2002) and this general impression is reinforced in Fig. 4; almost

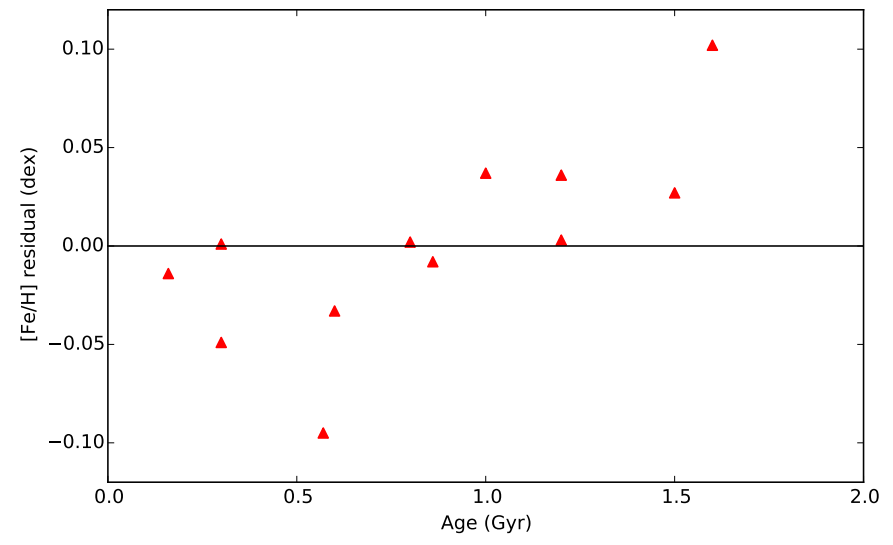

Fig. 5. Residuals in $[\mathrm{Fe} / \mathrm{H}]$ from the linear gradient as a function of cluster age.

all the younger clusters, with ages $<2$ Gyr, have lower metallicities than predicted in the model. Conversely, the oldest of the clusters lie at higher metallicities than predicted.

Netopil et al. (2016), investigating possible age-metallicity relationships among their homogenized literature sample, found evidence, in fact, of an increase in metallicity with age. Comparing metallicities of clusters with ages less than 0.5 Gyr to those with ages between 1 and $2.5 \mathrm{Gyr}$, corrected for the radial gradient, they found that the younger group had metallicities $\sim 0.07$ dex lower than the older group. This held even when they only considered clusters within $7 \leq R_{\mathrm{GC}} \leq 9 \mathrm{kpc}$. We can carry out the same exercise with the GES clusters, and find an identical result. Residuals from the linear gradient show a clear correlation with cluster age (Fig. 5); the younger clusters are at systematically lower metallicities, while the older ones are at systematically higher metallicities. Using the same age groupings adopted by Netopil et al. (2016), we find, as they did, that clusters with ages less than 0.5 Gyr show a mean residual of $-0.021 \pm 0.026$ while clusters older than 1.0 Gyr have a mean residual of $+0.041 \pm 0.037$. That this trend is evident in our homogeneously analyzed (and smaller) cluster sample that extends to $R_{\mathrm{GC}} \sim 5.5 \mathrm{kpc}$ is worth emphasizing.

As Netopil et al. (2016) point out, this increase in metallicity with age, contrary to what would be expected from simple expectations of chemical evolution, can be explained by the effect of radial migration, as more metal-rich and older objects from the inner disk move outward. An analogous result has been seen in a comparison of metallicity gradients traced by planetary nebulae and H II regions in M 31: the older nebulae exhibit a flatter gradient and higher oxygen abundances than the younger H II regions (Magrini et al. 2016). In their comparison to the chemical evolution model of Minchev et al. (2013), Netopil et al. (2016) found that the trend of increasing metallicity with age was qualitatively consistent with the effect of radial migration, but was stronger than predicted by the model. The same holds true for our cluster sample.

However, in the larger context, the exact magnitude and effect of radial migration on the chemical abundance trends seen in the Milky Way have yet to be firmly established. Observationally, evidence appears to be growing that radial migration effects cannot be ignored. The lack of an age-metallicity relation for stars in the solar neighborhood can be naturally explained by migration processes, for example (e.g., Haywood 2006). Also, the shapes of metallicity distribution functions in different radial bins of APOGEE red giant stars led Hayden et al. (2015) to conclude that "migration is of global importance in the evolution of 
the disk" (p. 12). Furthermore, some radial migration models can reproduce observations remarkably well (e.g., Loebman et al. 2016). However, radial migration models still vary from one to another on the assumption of initial conditions, as well as how the bar and spiral arms system are treated. Still more assumptions are made when a chemical evolution model is applied to a dynamical one (Minchev et al. 2013, are clear on the sensitivity of their results to assumptions made in their model). Adding chemistry to a dynamical model introduces additional uncertainties that complicate comparison of models to one another and to observations.

As a result, different models can produce conflicting predictions. For example, Minchev et al. (2013) found that the Sun most likely formed near somewhere between $R_{\mathrm{GC}}=5.5-7 \mathrm{kpc}$, while Martínez-Barbosa et al. (2015) found the probability that the Sun formed inside $R_{\mathrm{GC}}=8 \mathrm{kpc}$ to be no higher than $30 \%$. Furthermore, they found that when radial migration effects were strong, the Sun was more likely to migrate from the outer disk, and this only occurred in specific cases.

In an analysis of FGK stars near the Sun, Haywood et al. (2013) argue for the lack of a detectable influence of radial migration, at least in the solar neighborhood. They argue that the ellipticity of stellar orbits, plus the Sun's nearness to the transition to the metal-poor outer disk $\left(R_{\mathrm{GC}} \sim 10 \mathrm{kpc}\right)$, are sufficient to explain the presence of both metal-poor and metal-rich stars in the solar neighborhood. They furthermore argue that the Milky Way disk likely formed outside-in, rather than inside-out, based on the different age distributions of the metal-poor and metal-rich stars near the Sun.

Finally, it is still uncertain whether the effect of radial migration on open clusters is the same as that for field stars. Migration has been proposed to explain the presence of clusters with $[\mathrm{Fe} / \mathrm{H}] \sim+0.4$ dex near $R_{\mathrm{GC}} \sim 7 \mathrm{kpc}$, such as NGC 6791; however, a study by Jílková et al. (2012) concluded it was very unlikely. On the other hand, Fujii \& Baba (2012) used $N$-body simulations of clusters in a Galactic disk and found a timescale of $\sim 100 \mathrm{Myr}$ for the radial migration of open clusters. In that time period, they demonstrated a cluster could move $\sim 1.5 \mathrm{kpc}$ from its birth location, with minimal mass loss. However, their simulation did not include the effects of the bar, and so it is not clear whether their general conclusions apply to clusters inside the Solar circle, as we consider here. Martinez-Medina et al. (2016) demonstrated that spiral arms can lift open clusters $>1 \mathrm{kpc}$ above the Galactic mid-plane, which can explain the tendency of older $(>1 \mathrm{Gyr})$ clusters to have large $z$-distances, but they do not explore variations of cluster $R_{\mathrm{GC}}$ in their model.

In conclusion, to fully gauge the impact of radial migration on the formation and evolution of the disk, the full range of observations of different stellar populations at different locations in the disk (correcting for observational biases) must be compared with multiple chemo-dynamical models that are built upon different assumptions and have different uncertainties. This should be possible within the next decade, after the many ongoing and planned spectroscopic surveys of the Galaxy are complete.

\section{Summary}

We have presented an analysis of the inner disk metallicity gradient based on metallicities of 12 intermediate-aged clusters observed as part of the Gaia-ESO Survey. Our sample triples the number of clusters inside $R_{\mathrm{GC}}=7 \mathrm{kpc}$ compared to previous homogeneous literature studies, and also spans a wider range in age $(\sim 1.5 \mathrm{Gyr})$ than other studies of objects in this part of the disk (e.g., Cepheids in Andrievsky et al. 2002).

The metallicity gradient of our sample, which spans $5.5<$ $R_{\mathrm{GC}}<8 \mathrm{kpc}$, is $-0.10 \pm 0.02 \mathrm{dex} \mathrm{kpc}^{-1}$, consistent within uncertainties to the gradient measured by APOGEE red giant stars (Hayden et al. 2014). As our sample stars all lie within $150 \mathrm{pc}$ of the Galactic plane, the impact of a vertical metallicity gradient on our results is expected to be less than $0.05 \mathrm{dex}$, and would only change the radial gradient by $0.01 \mathrm{dex} \mathrm{kpc}{ }^{-1}$. The metallicity gradient shown by our inner disk sample is also consistent with that shown by the larger cluster sample of Netopil et al. (2016) in the range of $6<R_{\mathrm{GC}}<14 \mathrm{kpc}$. Therefore, the GES clusters do not support previous claims in the literature that the metallicity gradient steepens inside the solar circle.

We have also found that the GES clusters exhibit a trend of increasing metallicity with cluster age: after correcting for the effect of the gradient, clusters older than 1 Gyr are 0.06 dex more metal-rich than clusters aged $<0.5$ Gyr. Two clusters that exemplify this effect, Berkeley 44 (age $1.6 \mathrm{Gyr},[\mathrm{Fe} / \mathrm{H}]=+0.17 \pm$ 0.04 ) and NGC 4815 (age $570 \mathrm{Myr},[\mathrm{Fe} / \mathrm{H}]=-0.03 \pm 0.06$ ), both reside at $R_{\mathrm{GC}}=6.9 \mathrm{kpc}$, indicating that the dispersion in $[\mathrm{Fe} / \mathrm{H}]$ with $R_{\mathrm{GC}}$ is $\sim 0.2$ dex.

A comparison of the inner disk metallicity gradient to the predictions of the chemical evolution model of Minchev et al. (2014) found relatively poor agreement (Fig. 4). The agemetallicity relation for our sample is consistent with the effects of radial migration predicted by Minchev et al. (2014), as also discussed in Netopil et al. (2016), but the slope and temporal evolution of the gradient predicted by the model is at odds with the values shown by open clusters in general. We look forward to a comparison of cluster and model results when the Gaia-ESO Survey is complete.

Acknowledgements. The authors thank the referee for comments that improved the presentation of this research. This work was partly supported by the European Union FP7 programme through ERC grant number 320360 and by the Leverhulme Trust through grant RPG-2012-541. We acknowledge the support from INAF and Ministero dell' Istruzione, dell' Università e della Ricerca (MIUR) in the form of the grant "Premiale VLT 2012" and "The Chemical and Dynamical Evolution of the Milky Way and Local Group Galaxies" (prot. 2010LY5N2T). The results presented here benefit from discussions held during the Gaia-ESO workshops and conferences supported by the ESF (European Science Foundation) through the GREAT Research Network Programme. F.J.E. acknowledges financial support from the ARCHES project (7th Framework of the European Union, n 313146). S.V. gratefully acknowledges the support provided by Fondecyt reg. 1130721. U.H. acknowledges support from the Swedish National Space Board (SNSB). D.G. gratefully acknowledges support from the Chilean BASAL Centro de Excelencia en Astrofísica y Tecnologías Afines (CATA) grant PFB-06/2007.

\section{References}

Ahn, C. P., Alexandroff, R., Allende Prieto, C., et al. 2014, ApJS, 211, 17 Andreuzzi, G., Bragaglia, A., Tosi, M., \& Marconi, G. 2011, MNRAS, 412, 1265 Andrievsky, S. M., Bersier, D., Kovtyukh, V. V., et al. 2002, A\&A, 384, 140 Balcells, M. 2014, EAS Pub. Ser., 67, 227

Bergemann, M., Ruchti, G. R., Serenelli, A., et al. 2014, A\&A, 565, A89 Bird, J. C., Kazantzidis, S., \& Weinberg, D. H. 2012, MNRAS, 420, 913 Bonatto, C., Kerber, L. O., Bica, E., \& Santiago, B. X. 2006, A\&A, 446, 121 Borissova, J., Chené, A.-N., Ramírez Alegría, S., et al. 2014, A\&A, 569, A24 Bragaglia, A., Sestito, P., Villanova, S., et al. 2008, A\&A, 480, 79 Bressan, A., Marigo, P., Girardi, L., et al. 2012, MNRAS, 427, 127 Cantat-Gaudin, T., Vallenari, A., Zaggia, S., et al. 2014, A\&A, 569, A17 Cantat-Gaudin, T., Donati, P., Vallenari, A., et al. 2016, A\&A, 588, A120 Carraro, G., Janes, K. A., Costa, E., \& Méndez, R. A. 2006, MNRAS, 368, 1078 Carraro, G., Geisler, D., Villanova, S., Frinchaboy, P. M., \& Majewski, S. R. 2007, A\&A, 476, 217

Carrera, R., \& Pancino, E. 2011, A\&A, 535, A30

Cescutti, G., Matteucci, F., François, P., \& Chiappini, C. 2007, A\&A, 462, 943 
Chiappini, C., Matteucci, F., \& Gratton, R. 1997, ApJ, 477, 765

Chiappini, C., Matteucci, F., \& Romano, D. 2001, ApJ, 554, 1044

Cirasuolo, M., Afonso, J., Bender, R., et al. 2011, The Messenger, 145, 11

Clem, J. L., Landolt, A. U., Hoard, D. W., \& Wachter, S. 2011, AJ, 141, 115

Cui, X.-Q., Zhao, Y.-H., Chu, Y.-Q., et al. 2012, Res. Astron. Astrophys., 12, 1197

Cunha, K., Frinchaboy, P. M., Souto, D., et al. 2016, Astron. Nachr., in press [arXiv: 1601.03099]

Dalton, G., Trager, S. C., Abrams, D. C., et al. 2012, in Ground-based and Airborne Instrumentation for Astronomy IV, Proc. SPIE, 8446, 84460P

de Jong, R. S., Barden, S., Bellido-Tirado, O., et al. 2014, in Ground-based and Airborne Instrumentation for Astronomy V, Proc. SPIE, 9147, 91470M

De Silva, G. M., Freeman, K. C., Bland-Hawthorn, J., et al. 2015, MNRAS, 449, 2604

Donati, P., Beccari, G., Bragaglia, A., Cignoni, M., \& Tosi, M. 2014a, MNRAS, 437, 1241

Donati, P., Cantat Gaudin, T., Bragaglia, A., et al. 2014b, A\&A, 561, A94

Donati, P., Bragaglia, A., Carretta, E., et al. 2015, MNRAS, 453, 4185

Frasca, A., Biazzo, K., Lanzafame, A. C., et al. 2015, A\&A, 575, A4

Friel, E. D., Janes, K. A., Tavarez, M., et al. 2002, AJ, 124, 2693

Friel, E. D., Donati, P., Bragaglia, A., et al. 2014, A\&A, 563, A117

Frinchaboy, P. M., Thompson, B., Jackson, K. M., et al. 2013, ApJ, 777, L1

Froebrich, D., Scholz, A., \& Raftery, C. L. 2007, MNRAS, 374, 399

Fujii, M. S., \& Baba, J. 2012, MNRAS, 427, L16

Genovali, K., Lemasle, B., Bono, G., et al. 2013, A\&A, 554, A132

Genovali, K., Lemasle, B., Bono, G., et al. 2014, A\&A, 566, A37

Gilmore, G., Randich, S., Asplund, M., et al. 2012, The Messenger, 147, 25

Hayden, M. R., Holtzman, J. A., Bovy, J., et al. 2014, AJ, 147, 116

Hayden, M. R., Bovy, J., Holtzman, J. A., et al. 2015, ApJ, 808, 132

Haywood, M. 2006, MNRAS, 371, 1760

Haywood, M., Di Matteo, P., Lehnert, M. D., Katz, D., \& Gómez, A. 2013, A\&A, 560, A109

Heiter, U., Soubiran, C., Netopil, M., \& Paunzen, E. 2014, A\&A, 561, A93

Hill, V., Babusiaux, C., Gómez, A., et al. 2012, in EpJ Web Conf., 19, 6001

Jacobson, H. R., Pilachowski, C. A., \& Friel, E. D. 2011, AJ, 142, 59

Janes, K. A. 1979, ApJS, 39, 135

Janes, K. A., \& Hoq, S. 2011, AJ, 141, 92

Jeffries, R. D., Totten, E. J., Harmer, S., \& Deliyannis, C. P. 2002, MNRAS, 336, 1109

Jílková, L., Carraro, G., Jungwiert, B., \& Minchev, I. 2012, A\&A, 541, A64

Kubryk, M., Prantzos, N., \& Athanassoula, E. 2013, MNRAS, 436, 1479

Loebman, S. R., Roškar, R., Debattista, V. P., et al. 2011, ApJ, 737, 8

Loebman, S. R., Debattista, V. P., Nidever, D. L., et al. 2016, ApJ, 818, L6

Magrini, L., Sestito, P., Randich, S., \& Galli, D. 2009, A\&A, 494, 95

Magrini, L., Randich, S., Zoccali, M., et al. 2010, A\&A, 523, A11

Magrini, L., Randich, S., Romano, D., et al. 2014, A\&A, 563, A44

Magrini, L., Randich, S., Donati, P., et al. 2015, A\&A, 580, A85

Magrini, L., Coccato, L., Stanghellini, L., Casasola, V., \& Galli, D. 2016, A\&A 588, A91

Majewski, S. R., Schiavon, R. P., Frinchaboy, P. M., et al. 2015, AJ, submitted [arXiv: 1509.05420]

Martin, R. P., Andrievsky, S. M., Kovtyukh, V. V., et al. 2015, MNRAS, 449, 4071

Martínez-Barbosa, C. A., Brown, A. G. A., \& Portegies Zwart, S. 2015, MNRAS, 446, 823

Martinez-Medina, L. A., Pichardo, B., Moreno, E., Peimbert, A., \& Velazquez, H. 2016, ApJ, 817, L3

Mikolaitis, S.., Hill, V., Recio-Blanco, A., et al. 2014, A\&A, 572, A33

Minchev, I., Chiappini, C., \& Martig, M. 2013, A\&A, 558, A9

Minchev, I., Chiappini, C., \& Martig, M. 2014, A\&A, 572, A92

Netopil, M., Paunzen, E., Heiter, U., \& Soubiran, C. 2016, A\&A, 585, A150

Pancino, E., Carrera, R., Rossetti, E., \& Gallart, C. 2010, A\&A, 511, A56

Piatti, A. E., Clariá, J. J., Bica, E., Geisler, D., \& Minniti, D. 1998, AJ, 116, 801

Prisinzano, L., Damiani, F., Micela, G., et al. 2016, A\&A, 589, A70

Randich, S., Gilmore, G., \& Gaia-ESO Consortium. 2013, The Messenger, 154, 47

Recio-Blanco, A., de Laverny, P., Kordopatis, G., et al. 2014, A\&A, 567, A5

Roškar, R., Debattista, V. P., Quinn, T. R., Stinson, G. S., \& Wadsley, J. 2008, ApJ, 684, L79

Sacco, G. G., Morbidelli, L., Franciosini, E., et al. 2014, A\&A, 565, A113

Schönrich, R., \& Binney, J. 2009, MNRAS, 396, 203

Smiljanic, R., Korn, A. J., Bergemann, M., et al. 2014, A\&A, 570, A122

Smiljanic, R., Romano, D., Bragaglia, A., et al. 2016, A\&A, 589, A115

Stanghellini, L., \& Haywood, M. 2010, ApJ, 714, 1096
Steinmetz, M., Zwitter, T., Siebert, A., et al. 2006, AJ, 132, 1645

Sung, H., Bessell, M. S., Lee, B.-W., \& Lee, S.-G. 2002, AJ, 123, 290

Tosi, M. 1982, ApJ, 254, 699

Twarog, B. A., Ashman, K. M., \& Anthony-Twarog, B. J. 1997, AJ, 114, 2556

Yanny, B., Rockosi, C., Newberg, H. J., et al. 2009, AJ, 137, 4377

Yong, D., Carney, B. W., \& Teixera de Almeida, M. L. 2005, AJ, 130, 597

Yong, D., Carney, B. W., \& Friel, E. D. 2012, AJ, 144, 95

York, D. G., Adelman, J., Anderson, Jr., J. E., et al. 2000, AJ, 120, 1579

Zhang, B., Chen, X.-Y., Liu, C., et al. 2015, Res. Astron. Astrophys., 15, 1197

Zhao, G., Chen, Y.-Q., Shi, J.-R., et al. 2006, Chin. J. Astron. Astrophys., 6, 265

1 Massachusetts Institute of Technology, Kavli Institute of Astrophysics \& Space Research, Cambridge, MA, USA

e-mail: hrj@mit.edu

2 Department of Astronomy, Indiana University, Bloomington, IN, USA

3 Leiden Observatory, PO Box 9513, 2300 RA Leiden, The Netherlands

4 INAF-Osservatorio Astrofisico di Arcetri, Largo E. Fermi 5, 50125 Florence, Italy

5 INAF-Osservatorio Astronomico di Bologna, via Ranzani 1, 40127 Bologna, Italy

6 INAF-Osservatorio Astronomico di Padova, Vicolo Osservatorio 5, 35122 Padova, Italy

7 Dipartimento di Fisica e Astronomia, via Ranzani 1, 40127 Bologna, Italy

8 Dipartimento di Fisica e Astronomia, Universita di Padova, vicolo Osservatorio 3, 35122 Padova, Italy

9 Department for Astrophysics, Nicolaus Copernicus Astronomical Center, ul. Rabiańska 8, 87-100 Toruń, Poland

10 European Southern Observatory, Alonso de Cordova 3107 Vitacura, Santiago de Chile, Chile

11 Institute of Theoretical Physics and Astronomy, Vilnius University, A. Gostauto 12, 01108 Vilnius, Lithuania

12 Centro de Estudios de Física del Cosmos de Aragón (CEFCA), Plaza San Juan 1, 44001 Teruel, Spain

13 Departamento de Astronomía, Casilla 160-C, Universidad de Concepción, Concepción, Chile

14 Centro de Astrobiología (INTA-CSIC), Dpto. de Astrofísica, PO Box 78, 28691 Villanueva de la Cañada, Madrid, Spain

15 Suffolk University, Madrid Campus, C/ Valle de la Viña 3, 28003 Madrid, Spain

16 Institute of Astronomy, University of Cambridge, Madingley Road, Cambridge CB3 0HA, UK

17 Instituto de Astrofísica de Andalucía-CSIC, Apdo. 3004, 18080 Granada, Spain

18 Lund Observatory, Department of Astronomy and Theoretical Physics, Box 43, 22100 Lund, Sweden

19 INAF-Osservatorio Astronomico di Palermo, Piazza del Parlamento 1, 90134 Palermo, Italy

20 Department of Physics and Astronomy, Uppsala University, Box 516, 75120 Uppsala, Sweden

21 ASI Science Data Center, via del Politecnico SNC, 00133 Roma, Italy

22 Laboratoire Lagrange (UMR7293), Université de Nice Sophia Antipolis, CNRS, Observatoire de la Côte d'Azur, CS 34229, 06304 Nice Cedex 4, France

23 Astrophysics Research Institute, Liverpool John Moores University, 146 Brownlow Hill, Liverpool L3 5RF, UK

24 Departamento de Ciencias Fisicas, Universidad Andres Bello, Republica 220, Santiago, Chile

25 Instituto de Astrofísica e Ciências do Espaço, Universidade do Porto, CAUP, Rua das Estrelas, 4150-762 Porto, Portugal

26 Moscow MV Lomonosov State University, Sternberg Astronomical Institute, 119992 Moscow, Russia 